\title{
Basic Foundations of Clinical Electrophysiology
}

\author{
Andrew L. Wit, Ph.D.* \\ Department of Pharmacology, Columbia University College of Physicians and Surgeons, New York, New York, USA
}

\section{Key Words}

\section{Electrophysiology-Basic • Electrophysiology-Clinical}

Copyright $\odot 2015$ Science International Corp.

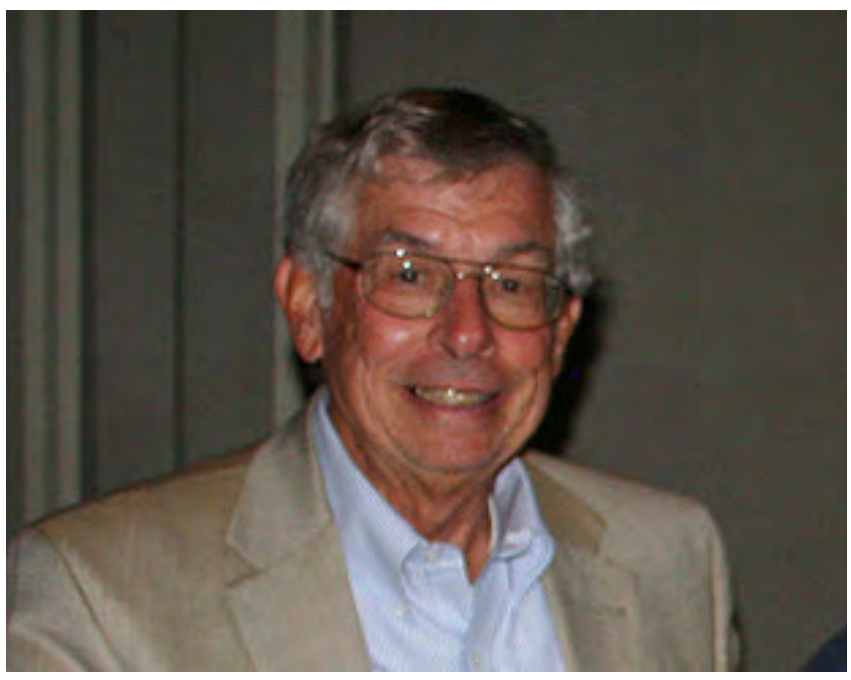

This section will be a continuing feature of the journal. As the title indicates, it will be composed of a series of articles designed to describe and teach basic electrophysiological concepts underlying clinical electrophysiology. In each issue, the column will elucidate the underlying electrophysiology and how it is translated into the electrophysiological characteristics of one of the case reports in a "user friendly" format. For example, mechanisms of arrhythmias and reasons for response to interventions, mechanisms for conduction abnormalities, mechanisms of effects of drugs, effects of electrical stimulation, and other interventions will be explored as they relate to the clinical case.

Translations from basic to clinical electrophysiology are usually based on properties described in vitro in the basic science lab, which are mimicked in vivo. To take one example, the response of isolated, superfused cardiac cells and tissues to electrical stimulation provides the foundation for understanding the effects of stimulation on clinical arrhythmias. Why are some arrhythmias initiated and terminated and others not? What is stimulation doing to the mechanism causing the arrhythmia, to the electrophysiological properties of the cells causing the arrhythmia? How does cellular electrophysiology enable different mechanisms of arrhythmias to be distinguished one from another by analyzing the effects of electrical stimulation?

Our philosophy is that clinical electrophysiologists need to have a solid foundation of basic electrophysiological knowledge to enable them to understand the causes and response to therapeutic interventions of cardiac arrhythmias. Not only will it help in the treatment of current patients, but it is an absolutely essential requirement for the continued development of this field. It was only because the founding fathers had this knowledge that the discipline has advanced to this stage. Without their intense interests in basic electrophysiology and its application to clinical arrhythmias, the clinical electrophysiology that we know today would not exist. They learned their basic electrophysiology by doing time in an experimental research laboratory, poking microelectrodes into cardiac cells to record (c) 2015 Arrhythmia Grand Rounds

Published by Science International Corp. ISSN 2326-4012

Accessible online at: http://arrhythmiagr.com/
* Corresponding Author:

Andrew L. Wit, Emeritus Professor of Pharmacology Department of Pharmacology

Columbia University College of Physicians and Surgeons

630 W 168th Street, New York, New York 10032, USA

Tel. +1 312819 1269, E-Mail: alw4@columbia.edu 
transmembrane potentials, and designing and studying the electrophysiology of arrhythmia models in hearts of mice, rabbits, cats, dogs, etc. From these experiences, the impetus arose to attempt to record the His bundle electrogram in man after it had been recorded in canine hearts by Brian Hoffman in the 1960 s, or to use programmed stimulation to evaluate AV junctional conduction in canine hearts and the mechanism of reentrant AV nodal tachycardia using dual AV nodal pathways in rabbit hearts from the experimental studies of Gordon Moe, around the same time. In parallel with these developments, cellular causes of impulse initiation such as automaticity and triggered activity and their properties were being studied with microelectrodes and translated to the clinical laboratory. The actuality of reentry as a cause of arrhythmias was debated well into the 1960s before laboratory experiments demonstrated its occurrence and proved its importance. The phenomenon of delayed after depolarization induced triggered activity was not translated into triggered clinical arrhythmias until the 1970s and 1980s. More recent laboratory studies have implicated early after depolarizations in the genesis of torsades de pointes associated with genetic and acquired long QT syndromes.

There are too many unsolved problems remaining for us not to take on the responsibility of ensuring that clinical cardiac electrophysiology keeps progressing and progress depends on a thorough understanding of underlying electrophysiological mechanisms. Despite all that has been learned over the past century, we should not become complacent that there is little more to know. I remember having a conversation with a well-known clinical electrophysiologist in the early 1970 's who indicated to me that now His bundle electrograms had been recorded under every conceivable circumstance of conduction disturbances and arrhythmias, there was not much more to do. Subsequent history speaks for itself. Modern therapies such as ablation of AV nodal reentrant tachycardia and pulmonary vein ablation were never even dreamed of at that time and would have seemed like science fiction if someone had proposed that these would become commonly used procedures. Improvements in the approach to catheter ablation of ventricular tachycardia through location of the isthmus region in a reentrant circuit, would have seemed far-fetched to the surgeons trying to prevent arrhythmias by aneurysectomy. Programmed electrical stimulation of the ventricles was strongly condemned by some until the mid to late 1970s. All of these procedures were developed through a thorough understanding of underlying electrophysiological mechanisms. That is the purpose of this column.

A more immediate practicality will be to review basic concepts that appear on the board exams in clinical electrophysiology. This basic electrophysiology that is pertinent to the clinical field is not easily accessible. It cannot be found in one place but must be searched out in numerous review articles and edited book chapters, often written for the basic or clinical research scientist and not for the clinician whose goal is to learn the fundamentals. When time is so valuable, most clinical electrophysiologists do not have the opportunity to search out all this necessary background information that had not been taught in any of the training environments that they have experienced. In this column we will present in a very understandable manner, these important concepts so that a collection of these articles will provide a comprehensive review of the basic electrophysiology necessary for understanding the underlying mechanisms of clinical electrophysiological abnormalities.

\section{Comment on this Article or Ask a Question}

Cite this article as: Wit AL. Basic Foundations of Clinical Electrophysiology. Arrhythmia Grand Rounds 2015;1(1): 36-38 DOI: http://dx.doi.org/10.12945/j.agr.2015.00010-14 